\title{
ÉDITORIAL
}

\section{Risque viral et eau d'alimentation}

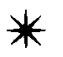

$\boldsymbol{S}^{t}$

L'EAU de boisson a de tous temps été à l'origine de manifestations épidémiques infectieuses relevant d'une étiologie fécale, il faut bien reconnaître que ce rôle propagateur d'importance maximale aux siècles derniers, se trouve réduit aujourd'hui à une valeur négligeable. En effet, l'étude des données épidémiologiques les plus récentes sur les épidémies de maladies virales à transmission hydrique fait apparaitre que seule l'hépatite à virus $A$ reste la virose où la contamination puisse sans contexte se faire par l'eau. Certes, les gastro-entérites-infectieuses (G.E.I.) d'origine hydrique sont les plus fréquentes, du moins aux U.S.A., mais leur étiologie virale n'est pas encore formellement démontrée. Enfin les adenovirus continuent à être responsables d'un bon nombre de conjonctivites des piscines. La plupart des poussées épidémiques rapportées (hépatite $A$ et G.E.I.) ont été associées à des systèmes d'eaux d'alimentation plus ou moins contaminés par des eaux fécalisées, ou mal contrôlés ou dont les procédés de désinfection étaient soit insuffisants, soit interrompus. On n'a jamais rapporté de manifestations épidémiques incriminant les enterovirus et ayant pour origine de l'eau de distribution publique considérée comme "potable».

Devant ce hiatus épidémiologique, trois hypothèses plausibles se présentent à l'esprit: ou bien la transmission par l'eau de ce groupe n'existe pas ; ou bien, si elle existe, son impact en santé publique est négligeable ; ou bien, tout tient dans l'insuffisance actuelle de nos moyens épidémiologiques permettant d'apporter une démonstration évidente de cette transmission.

Le risque entéroviral potentiel par l'eau existe et n'est pas niable. L'analyse de ses composantes, tout au long du cycle de l'eau : eaux usées $\longrightarrow$ eaux superficielles (de ruissellement) et souterraines $\longrightarrow$ eaux d'alimentation apporte la preuve irréfutable d'une présence virale, certes qualitativement et quantitativement variable, s'amenuisant au fur et à mesure qu'on avance dans le cycle, mais néanmoins démontrable. Existe-t-il une cassure dans le cycle, avec irruption d'eaux usées dans un des maillons, que le risque redevient maximal.

Il y a donc des raisons qui font qu'en dehors de toute épidémie caractérisée, il est difficile de donner une mesure exacte du risque hydrique entéroviral et de savoir pourquoi il s'exprime aussi mal épidémiologiquement.

A la réflexion, ces raisons sont multiples et intriquées et semblent être les suivantes :

1) les inconnues qui persistent quant à la notion de dose minimale infectante (D.M.I.) chez l'homme, surtout de ces virus «hydriques»;

2) les modalités d'expression de la D.M.I. dans une collectivité plus ou moins réceptive ou interviennent: l'éventail caractéristique des manifestations cliniques des enterovirus, le temps variable d'incubation, l'opposition classique entre entéroviroses-infections (la règle) et entéroviroses-maladies (l'exception), enfin le biais non négligeable que crée l'intervention de la voie prépondérante de transmission interhumaine qu'est la contamination féco-orale;

3) les données épidémiologiques très imprécises qui en découlent, conséquence de l'insuffisance de sensibilité de nos techniques épidémiologiques actuelles;

4) la double impossibilité de définir en termes numériques la concentration virale tolérable par unité de volume d'eau et d'arriver jamais à zéro particule dans l'élimination des virus hydriques. 


\title{
ÉDITORIAL
}

Des constatations précédentes découlent un certain nombre de sujets de réflexion et d'attitudes pratiques à prendre en considération.

1) Il convient, sans nul doute, d'avoir une notion plus exacte de l'existence, de la prévalence et de la concentration des virus hydriques pouvant se trouver dans l'eau potable, et tout en prenant conscience des possibilités et des limites des méthodes de concentration actuelles, de rechercher de meilleures techniques, plus fiables et plus sûres tant pour la récupération, que pour l'isolement et la quantification de tous les virus présents ( $y$ compris celui de l'hépatite $A$ et ceux présumés des G.E.I.).

Mais connaîtrait-on ces données, que le problème ne serait pas pour autant résolu puisqu'on ne dispose pratiquement d'aucune information valable sur la quantité de tel ou tel virus hydrique fécal (sauf peut-être le poliovirus) qui ingérée, est susceptible de déclencher l'infection chez l'homme. Il faudrait donc conjointement déterminer cette D.M.I. chez l'homme pour plusieurs virus entériques différents d'origine hydrique en sachant que ce genre de recherche pose de sérieux problèmes pratiques et déontologiques.

2) L'insuffisance de perception des méthodes épidémiologiques actuelles impose aussi d'essayer d'accroitre la probabilité de détection des cas de maladies isolés ou sporadiques, mais aussi des cas d'infection virale ayant l'eau pour point de départ, en mettant en æuvre des études prospectives bien structurées et bien conduites. Si une telle entreprise n'est pas insurmontable, il n'est pas certain cependant que ces études, très dispendieuses et qui coûteraient beaucoup de temps, apportent en fin de compte beaucoup d'informations complémentaires utiles en pratique.

3) Dans l'immédiat, il apparaît qu'il serait plus économique et sans doute plus rentable :

- Tout d'abord de s'attaquer au problème majeur, sinon prioritaire, que sont les eaux usées, quelle qu'en soit l'origine, et dont découlent tous les risques :

- en identifiant et en quantifiant, par tous les moyens disponibles et avec le souci de normalisation des méthodes, les virus présents dans ces eaux. Tous les efforts nécessaires pour isoler les virus non encore identifiés seront poursuivis ;

- en mettant tout en æuvre pour qu'au niveau des stations d'épuration (assainissement collectif et individuel) soient revus, sous l'angle de l'élimination et de l'inactivation des virus hydriques, les procédés jusqu'ici utilisés et qui n'ont pas été étudiés " a priori» dans cet objectif. A cet égard, il conviendrait de définir le traitement à appliquer pour que soit atteinte une réduction de l'ordre de $99,9 \%$ de la charge virale originelle. La stérilisation systématique d'un effluent ne paraît pas indispensable, sauf peut-être dans le cas du recyclage des eaux usées.

Dans cette évaluation, il devra davantage être tenu compte de ces deux importants facteurs que sont, d'une part la nécessité d'un échantillonnage véritablement représentatif de la charge influente et effluente et d'autre part, les difficultés inhérentes à la dissociation du complexe eaux usées - virus. Si des normes sont à promouvoir et à fixer, c'est à ce stade qu'elles doivent l'être en priorité, en les modulant avec le niveau de qualité du milieu récepteur et en fonction de certaines de ses caractéristiques (débit en particulier).

- Ensuite de poursuivre au niveau des eaux superficielles, où on peut encore déterminer sans trop de contingences techniques, même imparfaitement, la valeur de la charge virale, les recherches tendant à optimiser, en fonction de cette charge, le traitement séquentiel à leur appliquer. Lequel devrait avoir pour objectif prioritaire de préparer l'eau à une désinfection entraînant une inactivation virale, sûre, efficace et définitive. On s'efforcera en particulier de se placer davantage sur un plan industriel pour juger de la validité des conclusions tirées des diverses expériences de laboratoire sur la capacité des différentes méthodes utilisées pour éliminer ou inactiver les virus hydriques.

- Enfin de défnir dans la surveillance de l'eau d'alimentation un indicateur valable et de détection rapide des anomalies virologiques pouvant altérer ses qualités de potabilité.

4) Il y a peut-être aussi, dans la politique de l'eau, sous l'angle virologique et épidémiologique, une question de choix à faire, et dans une certaine rationalité économique, par des analyses de type coûts/avantages, de savoir s'il est préférable, par exemple, de tendre à la neutralisation des eaux usées et de pousser l'affinage des eaux de consommation ou de recourir à une vaccination efficace de la population consommatrice.

Telles sont les suggestions que l'on peut avancer après avoir fait un examen objectif des réalités épidémiologiques liées au risque viral par l'eau d'alimentation.

\author{
Professeur J.M FOLIGUET, \\ Biologiste des Hôpitaux
}

